\title{
Outcome of Percutaneous Iliosacral Screw Fixation of Sacroiliac Joint Disruptions
}

\author{
Mohamed El-sayed El-badawy*, El-sayed Abdel Moaty Mohamed, Ali Tawfik Alalfy, \\ Sherif Mohamed El-aidy
}

Department of Orthopedic Surgery, Faculty of Medicine, Zagazig University

*Corresponding author: Mohamed El-sayed El-badawy, Telephone: +201285203869, E-mail: dr_m.elbadawy@ yahoo.com

\begin{abstract}
Background: Closed reduction and percutaneous fixation of sacroiliac joint disruptions has the advantage of minimal invasion of compromised soft tissue, limited blood loss, decreased operation time, and decreased infection rates. The objectives of this study was to evaluate the functional and radiological outcome after percutaneous fixation of sacroiliac joint (SIJ) disruptions by cannulated screw.

Objectives: Management of sacroiliac joint disruptions by closed reduction and percutaneous fixation by iliosacral screw.

Patients and Methods: This study was carried out in Orthopedic Surgery Department, Zagazig University Hospitals, during the period from May 2018 to September 2019. The study included 12 patients with age ranged from 19-59 years, 7 males and 5 females. Mode of injury was road traffic accident in $58.3 \%$ of cases and fall from height in $41.7 \%$, right side was affected in 6 patients, left side was affected in 5 patient and one patient was affected bilaterally.

Results: All patients were evaluated clinically according to Majeed scoring system. The overall Majeed score was satisfactory in $75 \%$, while unsatisfactory results were in $25 \%$ of cases. Postoperative radiological evaluation was done according to Matta and tornetta. 25\% patients had excellent results, 50\% had good results and 25\% had fair results. Postoperative complication were superficial infection in 6 patients and one patient had chronic posterior pelvic pain.

Conclusion: Percutaneous method of fixation of the SIJ disruptions is effective and favourable method ensuring low operative time, high chance of obtaining stable pelvic ring with minimal blood loss, low incidence of complications and good rehabilitation results especially early mobilization.
\end{abstract}

Keywords: Pelvic Ring Injuries, Sacroiliac Joint Disruption, Iliosacral screw.

\section{INTRODUCTION}

The sacroiliac joint (SIJ) is formed by articulation between the auricular surface on the medial aspect of the ilium and lateral aspect of the sacrum supported by strong ligamentous complex anteriorly and posteriorly. The SIJ allowing transfer of forces from the upper body to lower part of the body and has two types of movement nutation and counter nutation (1). Sacroiliac joint disruptions usually is associated with pelvic ring injury. Pelvic ring injuries result from multiple forces either APC forces or LC forces or shearing forces or combined forces. Unstable pelvic injuries are usually associated with high rate of morbidity and mortality ${ }^{(2)}$.

The posterior SI complex is responsible for pelvic stability preventing anterior or posterior displacement of the axial skeleton over the pelvis. So, adequate assessment of polytraumatized patients with pelvic ring injuries and analysing pelvic stability should be done. Pain and limitation of function may follow SIJ instability ${ }^{(2,3)}$.

Different classifications were described for pelvic ring injuries depending on either the anatomic site of injury or the mechanism of injury or affection of weight bearing mechanism or pelvic stability. The most common and useful classifications for the diagnosis and management of the pelvic ring injuries are Tile classification depending on pelvic stability in vertical and horizontal planes and Young and Burgess classification depending on the mechanism of injury (4). The poly-traumatized patient represent a therapeutic challenge to the treating surgeon especially unstable pelvic injury due to high rate of mortality. The patient must have special care from the time of injury until stabilization. The treatment protocol of the American College of Surgeons in the Advanced Trauma Life Support (ATLS) Program is recommended for general assessment and evaluation of the patient ${ }^{(2,4)}$.

Evaluation of the patient with a pelvic injury begins with an accurate history of the incident. A full and detailed history should be taken from the patient or his accompanying persons whenever possible. Clinical evaluation of the patient either general or local evaluation, radiological assessment and neurovascular examination should be done for appropriate evaluation of patient with pelvic ring injury ${ }^{(5)}$.

Generally the objectives of treating patients with pelvic ring injury is to achieve union in satisfactory position, prevention of deformity and rehabilitation as soon as possible to ensure early return of mobility and function. Operative treatment of sacroiliac joint disruptions and pelvic injury is preferred ensuring optimum reduction and 
maintenance of fixation ${ }^{(6)}$. The treating modalities of SIJ disruptions included open methods of reduction, fixation and percutaneous fixation of the SIJ. Percutaneous fixation of the SIJ by screws is preferred as it has low incidence of complications especially wound infection, no blood loss and no time loss as compared to open method ${ }^{(6,7)}$.

Complications of SIJ disruptions include instability of pelvis, which may end in deformity, persistent SI pain and limitation of function, in addition to complications of trauma like haemorrhage, visceral and soft tissue injuries and thromboembolic complications from prolonged recumbency. The percutaneous iliosacral screw (ISS) may cause nerve root injury ${ }^{(2,7)}$.

\section{THE AIM OF WORK}

Evaluation of fixation of sacroiliac joint disruptions by percutaneous iliosacral screw.

\section{SUBJECTS AND METHODS}

The Site of study:

This study was conducted in Orthopedic Surgery

Department, Zagazig University Hospitals.

Sample size:

The study included 12 patients with sacroiliac joint disruptions either unilateral or bilateral.

\section{Inclusion criteria:}

1-Patients with sacroiliac joint disruptions indicated for surgery either isolated or associated with other injuries.

2-Surgically fit patients (males-females)

\section{Exclusion criteria:}

1. Ppatients younger than 18 years old.

2. Patients with old disruptions.

3. Surgically unfit patients.

4. Infection at surgical site

\section{Ethical Considerations:}

Informed consent was obtained from all participants and the study was approved by the Research Ethical Committee of Faculty of Medicine, Zagazig University.

\section{Methods:}

All patients were subjected to the following:

\section{History taking:}

Complete history was taken from all conscious and oriented patients. Age, sex, address, occupation and telephone number, mode of trauma, the affected side and associated medical problems.

\section{Clinical Examination:}

General examination: Every patient is examined and assessed according to the advanced trauma life support (ATLS):

After completion of primary survey, a secondary survey was started within 12-24 hours after injury. Complete examination from head to toe was performed with evaluation of complete history and presence of medical history allergies past illnesses.

\section{Assessment of associated injuries:}

Skeletal injuries: Non-skeletal injuries: urological, rectal, gynecological if applicable, muscle injury and breach to the skin overlying.

Vascular examination: Examination of the peripheral vasculature. Exclusion of DVT and compartmental syndrome

Neurological examination: Examination of affection of lumbosacral plexus mainly 14-15 nerve roots by examination of ankle and big toe dorsiflexion and examination of their sensory functions at their dermatomal level.

3-Radiological Evaluation: All patients are assessed radiologically by:

- Plain Radiograph.

AP radiographs, inlet and outlet views were obtained for the patients. They were used mainly to diagnose and classify the pelvic ring injury. Also, these views were assessed for the presence of associated bony injury e.g. acetabular fractures.

\section{- CT scan:}

Ct scan was obtained for all patients with axial, coronal, views and $3 \mathrm{D}$ views were done for better understanding of the pelvic lines. Post-operative CT scan is used for evaluation of reduction and accuracy of screw positioning.

\section{Treatment:}

This included the following:

\section{A-Initial treatment}

- External immobilization of the injured pelvis:

Was done through application of a pelvic binder or a circumferential wrapping with a bed sheet

\section{- Thromboembolic prophylaxis:}

- Encouragement of all patients to maintain active exercises for toes and ankle, whenever possible, for thromboembolic prophylaxis mechanically. Pneumatic calf and leg compression was used whenever available. Patients were kept well hydrated

- Low molecular weight heparin was given for 7 patients (had associated injuries that delay SIJ fixation) starting from the day of injury and stopped 12 hours before the operation. Low molecular weight heparin was given to all patients 6-12 hours postoperatively for 15 days.

- Catheterization of the urinary bladder

- IV cannulation.

\section{B-Operative treatment:}

- An informed consent was taken from every patient who has been involved in the study.

- Preoperative preparation of the colon using 
enema 24 hours before operation.

- Patients had either a dose of two grams of third generation cephalosporin at induction of anaesthesia.

- We used ordinary translucent table before starting, adjustment of the $\mathrm{C}$-arm was done to insure an accurate anteroposterior, inlet and outlet views needed for reduction and screw placement.

- Anesthesia: Spinal anaesthesia was given to 7 patients, epidural anaethesia to 4 patients and general anaethesia to one patient.

- Position: All patients were operated in supine position.

\section{- Preparations:}

Draping the entire lower abdomen free from the nipple line distally is performed, including the involved leg.

The following steps were followed to insert the iliosacral screw (s) percutaneously

- The C-arm of the image intensifier was positioned horizontally parallel to the transverse plane to the patient's pelvis with the concavity of the $\mathrm{C}$ arm under the operative table. The ilio-cortical density was determined on this view and the entry point of the guide wire was inferior to this mark.
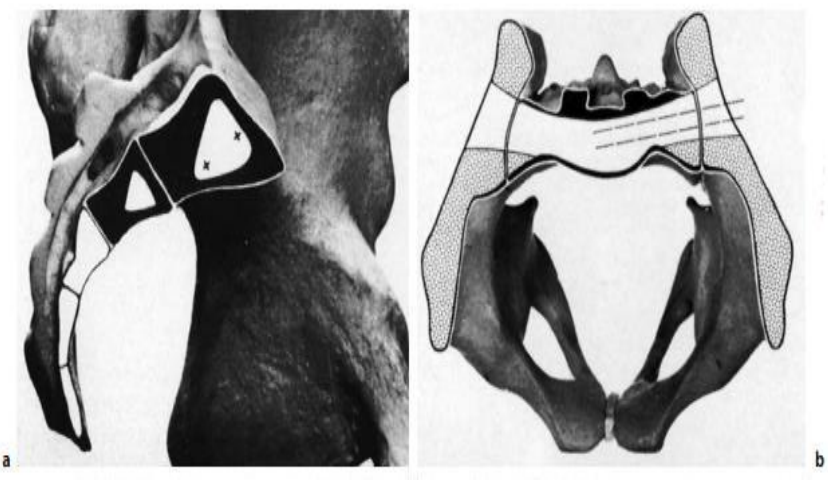

Fig. (1): (a) The lateral sacral view: anatomic model. (b) A cross section of the pelvis at the upper level of the S1 vertebral body shows the safest path for a screw at this level between the two dotted lines ${ }^{(8)}$.

- The superficial skin location for screw insertion is 2 $\mathrm{cm}$ posterior to the intersection of a line from the femoral shaft and a line dropped from the anterior superior iliac spine or by lateral view of the sacrum. The guide wire was inserted at the previously determined entry point on the lateral view and advanced by a power driver into the iliac cortex, Sacroiliac joint and the sacral cortex.

- Both inlet and outlet radiographs were obtained to check the planned trajectory of the guide wire. The guide wire should be above S1 foramen in the outlet anterior to the neural canal and not breaching the anterior sacral cortex in the inlet views.

- Measurement of the appropriate screw length was done over the guide wire.

- A $7.3 \mathrm{~mm}$ partially threaded screw was applied with washer and was tightened to compress the sacroiliac joint.

- A final check for the adequacy of the reduction and the screw position was done by intra-operative x-rays.

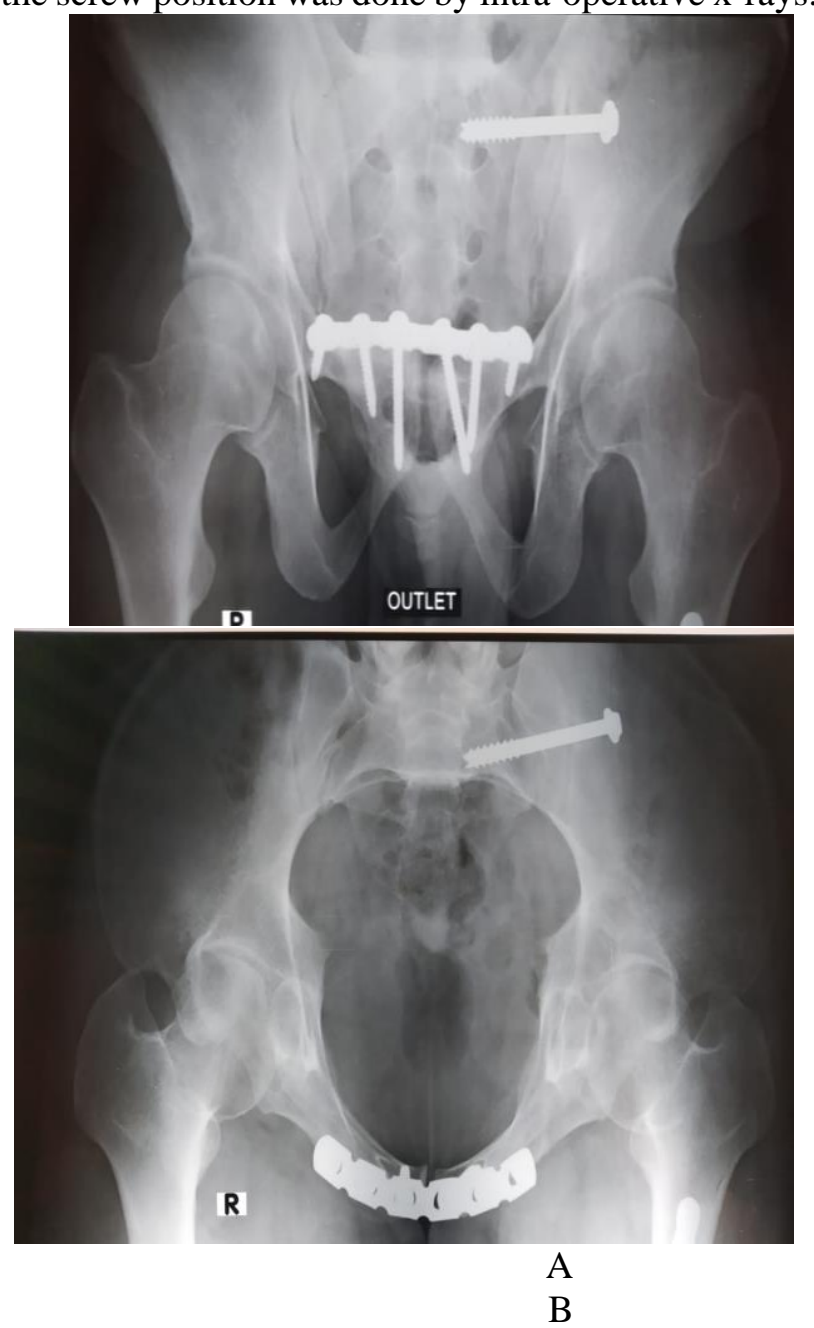

Fig. (2): Postoperative $X$-ray showing the final position of the Iliosacral screw (A) outlet view (B) inlet view.

\section{C-Post-operative:}

Discharge of patients was done 24 hours postoperatively for 5 patients. 7 patients were discharged 48 hours postoperatively after follow up by colleagues of other departments like general surgery and cardiothoracic departments.

\section{- Medications:}

1. Subcutaneous single injection of lowmolecular-weight heparin preparations (Clexane $40 \mathrm{IU}$ ) were used for prophylaxis against DVT which started 12 hours postoperatively lasting for at least 2 weeks.

2. Prophylactic parenteral broad spectrum antibiotics were used for 1 week, then oral antibiotics for 2 weeks.

3. Adequate analgesia

4. Postoperative $\mathrm{x}$-rays (AP view of the pelvis, inlet and outlet views) were done in addition to postoperative CT. These postoperative radiographs were used to assess reduction, 
degrees of displacement, quality of fixation and the presence of malpositioned screw.

\section{- Rehabilitation:}

The patients were mobilized from bed as early as possible. All patients, if their general condition allowed, were advised to start toe touch weight bearing with crutches after 6 weeks and then partial weight bearing after another 6 weeks. Patients started full weight bearing at 12 weeks postoperatively.

\section{- Radiological evaluation}

Post-operative AP radiographs were done routinely within the first postoperative week and before the patients were discharged from the hospital. Follow up radiographs were taken at 6 , 12 weeks and six months postoperatively. The radiographs were assessed for residual displacement and the quality of reduction. The posterior reduction was graded according to Matta and Tornetta ${ }^{(9)}$.

\section{Clinical evaluation:}

All patients were assessed according to Majeed scoring system at the end of the follow up period. The patients were interviewed by the examining surgeon to be asked some questions and their answers were scored according to majeed.

\section{Statistical analysis}

Recorded data were analyzed using the statistical package for social sciences, version 20.0 (SPSS Inc., Chicago, Illinois, USA). Quantitative data were expressed as mean \pm standard deviation (SD). Qualitative data were expressed as frequency and percentage. The following tests were done:

- Independent-samples t-test of significance was used when comparing between two means.

- Chi-square $\left(\mathrm{x}^{2}\right)$ test of significance was used in order to compare proportions between two qualitative parameters.

- The confidence interval was set to $95 \%$ and the margin of error accepted was set to $5 \%$. The $\mathrm{p}$ value was considered significant as the following:
- P-value $<0.05$ was considered significant.

- P-value $<0.001$ was considered as highly significant.

- P-value >0.05 was considered insignificant.

\section{RESULTS}

This study include twelve patient of SIJ disruptions underwent surgery in Zagazig University Hospital with age ranged from 19-59 years, 7males and 5 females. Mode of injury was road traffic accident in $58.3 \%$ of cases and fall from height in $41.7 \%$, right side was affected in 6 patients, left side was affected in 5 patients and one patient was affected bilaterally (Table 1).

Table (1): Descriptive statistics of the twelve patient of the study.

\begin{tabular}{|l|l|l|}
\hline & Range & Mean \\
\hline Age & $19-59$ patients & 39.0 \\
\hline $\begin{array}{l}\text { Timing (lag } \\
\text { days) }\end{array}$ & $1-7$ days & 3.83 \\
\hline $\begin{array}{l}\text { Operation time } \\
\text { /minutes }\end{array}$ & $30-50$ minutes & 37.0 \\
\hline
\end{tabular}

According to tile classification Injuries of 5 patients $(41.7 \%)$ were classified B1and 2 patients (16.7\%) were B2. 5 patients $(41.7 \%)$ were C2. According to Young and Burgess classification, 2 patients $(16.7 \%)$ were APC1, 5 patients $(42.7 \%)$ Were APC2, vertical shear was in $2(16.7 \%)$ patients and 3 patients $(25 \%)$ were combined mechanism. The duration of follow-up ranged from 6 to 12 months with a mean of 9 months. All patients in this study were operated within time frame ranged from I day to 7 days with mean (3.83) days an SD (2.17) days. Operation time was minimum 30 minutes and maximum 50 minutes with mean 37 minutes (Table 1). The patients were assessed both clinically and radiologically at the end of the follow-up period.

able (3): Relation between radiological results and clinical results

\begin{tabular}{|c|c|c|c|c|c|c|c|}
\hline & \multicolumn{2}{|c|}{ Majeed } & \multirow[t]{2}{*}{ Total } & \multirow[t]{2}{*}{$\mathrm{X}^{2}$} & \multirow[t]{2}{*}{$\mathrm{P}$} \\
\hline & & & Satisfactory & Unsatisfactory & & & \\
\hline \multirow{6}{*}{$\begin{array}{c}\text { X-ray } \\
\text { evaluation }\end{array}$} & \multirow[t]{2}{*}{ Fair } & $\mathbf{N}$ & 0 & 2 & 2 & & \\
\hline & & $\%$ & $0.0 \%$ & $66.7 \%$ & $16.7 \%$ & & \\
\hline & \multirow[t]{2}{*}{ Good } & $\mathbf{N}$ & 8 & $\mathbf{0}$ & 8 & 9.33 & 0.009* \\
\hline & & $\%$ & $88.9 \%$ & $0.0 \%$ & $66.7 \%$ & & \\
\hline & \multirow[t]{2}{*}{ Excellent } & $\mathbf{N}$ & 1 & 1 & 2 & & \\
\hline & & $\%$ & $11.1 \%$ & $33.3 \%$ & $16.7 \%$ & & \\
\hline \multirow{2}{*}{\multicolumn{2}{|c|}{ Total }} & $\mathbf{N}$ & 9 & 3 & 12 & & \\
\hline & & $\%$ & $100.0 \%$ & $100.0 \%$ & $100.0 \%$ & & \\
\hline
\end{tabular}

\section{Clinical results:}

Patients of this study were subjected to clinical examination at the last follow up visit which was based on Majeed score. The final overall results were 
considered satisfactory in $9(75 \%)$ patients; $3(25 \%)$ were excellent, $6(50 \%)$ were good, and 3 patients (25\%) were unsatisfactory fair outcome (Table 2).

Table (2): Majeed score distribution among studied group.

\begin{tabular}{|l|l|c|c|}
\hline \multirow{3}{*}{ Majeed score } & Fair & $\mathrm{N}$ & $\%$ \\
\cline { 2 - 4 } & Good & $\mathbf{3}$ & $\mathbf{2 5 . 0}$ \\
\cline { 2 - 4 } & Excellent & $\mathbf{3}$ & $\mathbf{5 0 . 0}$ \\
\hline Overall & Satisfactory & $\mathbf{9}$ & $\mathbf{7 5 . 0}$ \\
\cline { 2 - 4 } Majeed score & $\begin{array}{l}\text { Unsatisfact } \\
\text { ory }\end{array}$ & $\mathbf{3}$ & $\mathbf{2 5 . 0}$ \\
\cline { 2 - 4 } & Total & $\mathbf{1 2}$ & $\mathbf{1 0 0 . 0}$ \\
\hline
\end{tabular}

Radiological results:

Our post-operative AP radiographs that assessed vertical displacement reduction by measuring amount of distraction between two femoral heads according to Matta and Tornetta 1996 showed the following results:

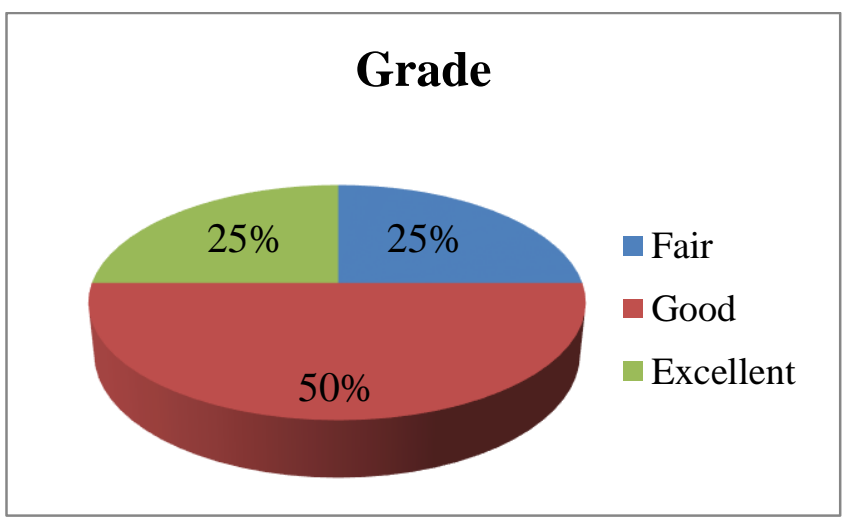

Fig. (3): Graph showing radiological grading among patients of the study.

Three patients were excellent with residual displacement less than $5 \mathrm{~mm}$.

Six patients were good with residual displacement from 5-10 mm.

Three patients were fair with residual is placement from 11-20 mm.

\section{Complications:}

Among the studied cases in this study no preoperative or intra operative complication were detected. All complications were reported postoperatively. $25 \%$ of patients reported superficial infection responding to drainage and repeated dressing and antibiotics. Only one patient had recurrent posterior pelvic pain improved with analgesia and anti-inflammatory medications.

\section{DISCUSSION}

Pelvic stability depends on an intact posterior sacroiliac complex. This complex can withstand the transference of the weight-bearing forces from the spine to the lower extremities ${ }^{(10)}$. Unstable pelvic ring injuries are serious condition, associated with a high rate of morbidity and mortality (11). Early surgical stabilization gave advantages of pain reduction, early mobilization of the patient and in the end ensures good functional recovery in the long term ${ }^{(3)}$.

Non operative treatment does not offer accepted reduction and limits the patient to bed with all possible complications ${ }^{(\mathbf{1 1}, \mathbf{1 2})}$. The purpose of this study was to describe the technique of percutaneous sacroiliac screw in cases of SIJ disruptions using conventional fluoroscopy and evaluate the perioperative complications and radiological results.

Comparing our study of percutaneous fixation of SIJ with other studies of fixation of the SIJ joint either by using percutaneous method or open reduction and plating of the SIJ or usage of spinopelvic fusion method showed difference in functional outcome, radiological outcome and complications which were found to be of higher incidence with open method.

Results of this study either clinical or radiological are near to results of other studies using the same technique. Schweitzer et al. ${ }^{(12)}$ in their study had satisfactory radiological outcome in $97.18 \%$ of patients and only $2.81 \%$ of patients had unsatisfactory radiological outcome. Also, satisfactory clinical results in $87,3 \%$ of patients and unsatisfactory in $7.14 \%$ with low incidence of complications. Lindahl and Hirvensalo ${ }^{(13)}$ had no poor radiological outcome. Excellent results in $65.34 \%$, good results in $24.75 \%$ and fair results in 9.9\%. Clinical results also were found near to results of this study. Satisfactory clinical results were found in $83.16 \%$ of patients and fair results in $15.84 \%$ and only $0.9 \%$ of patients had poor results. Abhishek et al. ${ }^{(14)}$ documented in their study that $51.22 \%$ of cases had excellent clinical outcome, $31.7 \%$ had good outcome and unsatisfactory results were fair in $9.7 \%$ of cases or poor in $7.31 \%$ of cases. These results are near to the results of our study also near in their low incidence of complications.

Open reduction and fixation of the sacroiliac joint (SIJ) allow visualization of the joint and exact reduction but the complications associated with the technique as documented in comparative study make the percutaneous method favorable technique for fixation of the sacroiliac joint. El Mannawy et al. ${ }^{(15)}$ documented the occurrence of deep tissue infection and foot drop and pelvic tilt in their study of anterior stabilization of the SIJ. Mardanpour and Rahbar ${ }^{(16)}$ documented various complications following open method of SIJ fixation including deep and superficial infections, injury to lateral cutaneous nerve of the thigh, pulmonary embolism and urinary tract infections. Simpson et al. ${ }^{(17)}$ in study of open reduction and fixation of SIJ either by staples or plate documented occurrence of loss of reduction in two patients with stable fixation and one patient with plate fixation. Sobhan et al. ${ }^{(18)}$ in study of spino-pelvic 
fixation in cases of sacroiliac joint injuries found satisfactory clinical results in $78 \%$ of cases and excellent reduction in $92.8 \%$ of cases but documented occurrence of implant failure in $7.1 \%$ and wound infection in $21.4 \%$ of case.

Complications associated with percutaneous method usually encountered in superficial infection or nerve root injury or malpositioning of the screw. The most serious complication is injury to lumbosacral roots due to misplacement of the screw and this risk increased with prescence of incomplete reduction. Several techniques used to avoid misplacement of the screw like CT guided and computer assisted navigation guided techniques but these methods have their limitation that lead to usage of fluoroscopy in majority of cases ${ }^{(\mathbf{1 9})}$.

\section{CONCLUSION}

Operative treatment is favourable method of treating SIJ injuries with maximum efficiency and limited complications. The goal of operative treatment is to preserve functional painless stable pelvic ring that can continue to function for the rest of the patient life. Early surgical intervention make reduction easier with high chance of anatomic reduction.

Percutaneous method of fixation of the SIJ disruptions is effective method of fixation of unstable pelvic ring injuries generally and SIJ disruptions especially ensuring low operative time, high chance of obtaining stable pelvic ring with minimal blood loss, low incidence of complications and good rehabilitation results especially early mobilization.

Conflict of interest: The authors declare no conflict of interest.

Funding sources: The authors had no funding to report

Acknowledgement: The authors are grateful for the patients without whom this study would not be done.

\section{REFERENCES}

1. Solonen KA (1957): The sacroiliac joint in the light of anatomical, roentgenological and clinical studies. Acta Orthop Scand., 27: 1-127.

2. Agarwal A (2015): Pelvic ring fractures. In: Rockwood and Green's fractures in adults. Court-Brown CM, Heckman JD, MCqueen M.M et.al. (eds). $8^{\text {th }}$ ed. Philadelphia, Wolter Kluwer Health, 4: 1795-1884.

3. Failinger MS, McGainty PL (1992): Current concepts review unstable fractures of the pelvic ring. J Bone Joint Surg., 74: 781-86.

4. Guyton JL, Perez A (2016): Fracture of acetabulum and pelvis in: Campbell's operative orthopaedics. Azar FM, Canale ST, Beaty JH, $13^{\text {th }}$ edition. e- book, Elsevier Health Sciences, 56: 2865-2912.

5. O'Brien PJ, Dickson KF (2005): Pelvic fractures evaluation and acute management. In: Orthopaedic knowledge update, Trauma 3. Rosemont, IL. AAOS.
https://www.aaos.org/OKOJ/vol15/issue9/OKOJ-15-9$1 /$ ?ssopc $=1$

6. Schweitzer D, Alejandro Z, Marcelo C et al. (2008): Closed reduction and iliosacral percutaneous fixation of unstable pelvic ring fractures. Injury, 39: 869-874.

7. Routt ML, Nork SE, Mills WJ (2000): Percutaneous fixation of pelvic ring disruptions. Clin Orthop Relat Res., 375: 15-29.

8. Routt ML, Meier MC, Kregor PJ et al. (1993): Percutaneous ilio-sacral screws with the patient supine technique. Oper. Techn. Orthop., 3: 35- 45.

9. Matta JM, Tornetta $P$ (1996): Internal fixation of unstable pelvic ring injuries. Clin Orthop Relat Res., 329: 129-140.

10. Tile M, Helfet D, Kellam J et al. (2003): Fractures of the Pelvis and Acetabulum. 3rd ed. Philadelphia: Lippincott Williams \& Williams, Pp: 12-21

11. Cole JD, Blum AD, Ansel LJ et al. (1996): Outcome after fixation of unstable posterior pelvic ring injuries. Clinical Orthopaedics Related Res., 329: 160179.

12. Schweitzer D, Zylberberg A, Cordora M et al. (2008): Closed Reduction and Percutaneous Fixation of Unstable Pelvic Ring Fractures. Elsevier Injury Journal, 39: 869874

13. Lindahl J, Hirvensalo E (2005): Outcome of Operatively Treated Type-C Injuries of Pelvic Ring. Act Orthopaedica, 76 (5): 667-678.

14. Abhishek SM, Azhar AL, Vijay GB et al. (2015): Functional Outcome with Percutaneous Ilio-sacral Screw Fixation for Posterior Pelvic Ring Injuries in Patients Involved in Heavy Manual Laboring. Malysean Orthop J., 3: 23-27.

15. El-Mannawy M, El Shoura SA, Youssef SA et al. (2015): Treatment of sacroiliac joint disruption with ant stabilization. Egypt Ortho J., 6: 45-50

16. Mardanpour K, Rahbar M (2013): The Outcome of Surgically Treaated Traumatic Un stable Pelvic Fracture by Open Reduction and Internal Fixation. J Inj \& Violence, 5 (2): 77-82.

17. Simpson L, Waddel J, Leighton $K$ et al. (1987): Anterior Approach and Stabilization of the Disrupted Sacroiliac Joint. J of Trauma, 12: 1332-1339.

18. Sobhan MR, Abrisham S, Vakili M et al. (2016): Spinopelvic Fixation of Sacroiliac Joint Fractures and Fracture-Dislocations: A Clinical 8 Years Follow-Up Study. ABJS., 4: 381-386.

19. El-Desouky II, Mohamed M, Kandil A (2016): Percutaneous iliosacral screw fixation in vertically un stable pelvic injuries, a refined conventional method. Acta Orthop Belg., 82: 52-59. 